\title{
Reshaping acetylated peptide selectivity between human BET Brd2 bromodomains BD-I and BD-II in glioblastoma by rationally grafting secondary anchor residues
}

\author{
Xifeng Fei, Anqi Wang, Xuan Meng and Zhimin Wang \\ Department of Neurosurgery, Kowloon Hospital, Shanghai Jiaotong University School of Medicine, Suzhou 215028, China
}

\begin{abstract}
Selective inhibition of BET Brd2 BD-I and BD-II bromodomains is expected to elicit subtle pharmacological difference in anti-glioblastoma therapy. Here, structural basis and energetic property underlying the selective interaction of acetylated peptide ligands with Brd2 BD-I and BD-II were investigated in detail using molecular simulation and computational analysis. It is revealed that the acetyl-lysine is, as expected, a primary anchor residue that confers affinity and stability to bromodomain-peptide binding, while few secondary anchor residues flanking the acetyl-lysine determine specificity and selectivity of peptide interaction with different bromodomains. We also demonstrated that peptide selectivity can be totally reversed by only grafting the secondary anchor residues from one to another. As an instance, fluorescence-based assays showed that the Stat3-derived acetylated peptide Stat3_K87 possesses a high affinity to BD-II $\left(K_{\mathrm{d}}{ }^{\mathrm{BD}-\mathrm{II}}=9.7 \mu \mathrm{M}\right)$ and a strong selectivity for BD-II over BD-I ( $S=0.21$-fold). Grafting the three secondary anchor residues Lys8, Gly11 and Gly13 of a BD-I-over-BD-II selective $\mathrm{H} 4 \mathrm{~N}$-terminal peptide to the corresponding residue positions of Stat3_K87, which results in a grafted counterpart Stat3(KGG)_K87, can completely change the peptide selectivity from the BD-II-over-BD-I ( $S=0.21$-fold) of Stat3_K87 to the BD-I-over-BD-II ( $S=2.5$-fold) of Stat3(KGG)_K87.
\end{abstract}

Key words: BET Brd2 - Bromodomain - Acetylated peptide - Peptide selectivity - Glioblastoma

\section{Introduction}

The bromodomains and extraterminal domain (BET) family of proteins are essential transcriptional regulators that control a variety of gene expressions and play key regulatory roles in cellular proliferation, cycle progression and apoptosis (Picaud et al. 2013). This family includes four members, i.e. $\operatorname{Brd} 2, \operatorname{Brd} 3, \operatorname{Brd} 4$ and Brdt; they are characterized by the presence of two conserved regions: the N-terminal bromodomains I and II (BD-I and BD-II) which bind acetylated chromatin, and the extraterminal (ET) domain whose function is unknown (Taniguchi 2016). The BET proteins are implicated in the development of many diverse diseases and,

Correspondence to: Zhimin Wang, Department of Neurosurgery, Kowloon Hospital, Shanghai Jiaotong University School of Medicine, Suzhou 215028, China

E-mail:wzhimin@hotmail.com zhiminwwang@sina.cn in recent years, BET bromodomains have been recognized as a new and attractive druggable target for cancer treatment (Jung et al. 2015; Padmanabhan et al. 2016).

The four members of BET family share a high sequence homology and structural conservation, but may induce divergent biological events in downstream cellular pathways (Deeney et al. 2016). Therefore, selective inhibition of BET proteins has been established as a versatile therapeutic strategy against distinct proliferative diseases (Filippakopoulos et al. 2010). Among the four BET members, Brd2 has been found to play a critical role in the pathogenesis of glioblastoma (GBM) and some other brain tumors. Pastori and coworkers demonstrated that the Brd2 RNA was significantly overexpressed in GBM and disruption of the expression considerably reduced GBM cell proliferation in vitro and in vivo (Pastori et al. 2014). Previously, a variety of smallmolecule BET inhibitors such as OTX015 and I-BET151 have been developed with antitumor effects alone and in combination with conventional therapies in GBM models 
(Long et al. 2014; Berenguer-Daizé et al. 2016). However, chemical agents generally exhibit broad specificity and high cross-reactivity due to the high structural conservation across the active sites of four BET members; animal models also revealed that nonselective BET inhibition would cause marked lymphoid and hematopoietic toxicity (Lee et al. 2016).

Instead of small-molecule chemical drugs, biologic peptide inhibitors that mimic the acetylated sites of BETinteracting partners have been recently found to possess high selectivity between Brd2 and Brd4 (Zhu et al. 2017). This is because peptide ligands are linear flexible molecules that do not only bind at the conserved active site of BET proteins, but also interact with other nonconserved regions out of the site. Usually, BET recognizes and binds at acetyl-lysine residue, called primary anchor residue. In addition, some other residues flanking the acetyl-lysine also contribute the recognition specificity of BET-peptide interaction; we called them secondary anchor residues. Selective inhibition of BD-I and BD-II in a single BET protein is expected to elicit subtle pharmacological difference in anti-GBM therapy due to the genetic diversity of this disease (Cheng et al. 2013). Here, we attempt to rationally graft the secondary anchor residues across different acetylated peptide ligands to reshape the peptide selectivity between the bromodomains BD-I and BD-II of Brd2.

\section{Materials and Methods}

\section{Acetylated peptide ligands of Brd2 BD-I and BD-II}

Human histone $\mathrm{H} 4$ is the primary binding target of BET Brd2 protein (Kanno et al. 2004). According to the crystal structure of human nucleosome core particle the $\mathrm{H} 4$ is tightly packed in the nucleosome and only its $\mathrm{N}$-terminal tail ( ${ }^{1}$ SGRGKGGKGLGKGGA ${ }^{15}$ ) is highly flexible and exhibits large intrinsic disorder (Tsunaka et al. 2005). The 15-residue tail contains three lysine residues, i.e. Lys5, Lys8 and Lys12, in which the Lys5 and Lys12 are tended to be acetylated and, then, recognized by Brd2 (Huang et al. 2007; Umehara et al. 2010a). The $\mathrm{N}$-terminal peptides acetylated at Lys5, Lys12 and both of them (H4_K5, H4_K12 and $\mathrm{H} 4$ K5 512 , respectively) as well as their binding affinities and cocrystallized structures with Brd2 BD-I and BD-II are listed in Table 1. Umehara et al. (2010b) also solved the crystal structure of Brd2 BD-I in complex with the acetylated core binding sequence $\mathrm{cH} 4$ K $12\left({ }^{6} \mathrm{GGKGLGK} K_{a c} \mathrm{GGA}^{15}\right)$ of $\mathrm{H} 4 \mathrm{~N}$-terminal tail (Table 1), where the whole sequence is tightly packed against the BD-I surface. In addition, Brd2 $\mathrm{BD}$-II has been reported to recognize and interact with the acetylated peptide Stat3_K87 $\left({ }^{281}\right.$ HNLLRIK $_{a c}$ QFLQS $\left.^{292}\right)$ of Stat3 to potentiate the transcriptional program for Th17 cell differentiation (Cheung et al. 2017) (Table 1). Recently, Zhu et al. (2017) demonstrated that this peptide can bind at Brd2 BD-II with a high affinity $\left(K_{\mathrm{d}}=5.9 \mu \mathrm{M}\right)$ and moderate selectivity for Brd2 over Brd4 (2.5-fold).

\section{Modeling the complex structures of Brd2 BD-I and BD-II with acetylated peptides}

The crystal/solution complex structures of Brd2 BD-I with acetylated peptides H4_K5, H4_K12, H4_K5K12 and cH4_K12 as well as Brd2 BD-II with acetylated peptides H4_K5K12 and Stat3_K87 are available in the RCSB protein data bank (PDB) database (Berman et al. 2000) (Table 1). The mutants of these peptides in complex with Brd2 BD-I and $\mathrm{BD}-\mathrm{II}$ can be readily modeled using a virtual mutagenesis strategy based on corresponding complex crystal structures. The strategy first manually removed the old side chains of mutated peptide residues and then automatically added new side chains to the residues by using BetaSCPWeb server (Ryu et al. 2016). Peptide ligand exchange between Brd2 BD-I and BD-II was modeled using a computational strategy (Bai et al. 2017). For instance, the solution complex structure of BD-II with peptide Stat3_K87 has recently been solved by Cheung

Table 1. The acetylated peptide ligands of Brd2 BD-I and BD-II

\begin{tabular}{|c|c|c|c|c|c|}
\hline \multirow{2}{*}{ Bromodomain } & \multicolumn{3}{|l|}{ Peptide } & \multirow{2}{*}{$\mathrm{PDB}^{b}$} & \multirow{2}{*}{$K_{\mathrm{d}}(\mu \mathrm{M})^{c}$} \\
\hline & Name & Sequence $^{a}$ & Origin & & \\
\hline \multirow{4}{*}{ BD-I } & H4_K5 (Umehara et al. 2010b) & ${ }^{1}$ SGRGK $_{a c}$ GGKGLGKGGA ${ }^{15}$ & Histone H4 & - & 1800 \\
\hline & H4_K12 (Umehara et al. 2010b) & ${ }^{1}$ SGRGKGGKGLGK ${ }_{a c} G_{G A}{ }^{15}$ & Histone H4 & 2DVQ & 930 \\
\hline & H4_K5K12 (Umehara et al. 2010b) & ${ }^{1} \mathrm{SGRGK}_{a c} \mathrm{GGKGLGK}_{a c} \mathrm{GGA}^{15}$ & Histone H4 & - & 360 \\
\hline & cH4_K12 (Umehara et al. 2010b) & ${ }^{6} \mathrm{GGKGLGK}_{a c} \mathrm{GGA}^{15}$ & Histone $\mathrm{H} 4$ & 2DVR & - \\
\hline \multirow{2}{*}{ BD-II } & H4_K5K12 (Umehara et al. 2010a) & ${ }^{1} \mathrm{SGRGK}_{a c} \mathrm{GGKGLGK}_{a c} \mathrm{GGA}^{15}$ & Histone $\mathrm{H} 4$ & $2 \mathrm{E} 3 \mathrm{~K}$ & - \\
\hline & Stat3_K87 (Cheung et al. 2017; Zhu et al. 2017) & ${ }^{281}{ }^{\text {HNLLRI }} K_{a c}$ QFLQS $^{292}$ & Stat3 & $5 \mathrm{US}$ & 5.9 \\
\hline
\end{tabular}

${ }^{a}$ acetyl-lysine residue; ${ }^{b}$ the RCSB PDB id of solved complex structure of Brd2 BD-I or BD-II with acetylated peptide; ${ }^{c}$ measured affinity of acetylated peptide binding to Brd2 BD-I or BD-II. 
et al. (2017), and we used it as template to model the complex structure of the peptide with BD-I. In the procedure, apo BD-I structure was superposed onto the BD-II-Stat3_K87 complex template to obtain a superposed system of BD-I/ BD-II/Stat3_K87, from which the BD-II was removed manually, resulting in the artificial complex structure of BD-I with Stat3_K87, which was then refined by using 3Drefine server (Bhattacharya et al. 2016) to eliminate bad atomic contacts and overlapping involved in the coarse-grained structure (Tian et al. 2011; Lou et al. 2015).

\section{Dynamics simulation and energetics calculation}

The molecular dynamics (MD) simulations of Brd2 BD-I and BD-II complexes with acetylated peptides were performed with amber ff03 force field (Duan et al. 2003) implemented in AMBER suite software (Case et al. 2005). The force field parameters for acetyl-lysine residue were taken from Khoury et al. (2013). The complex system was solvated in a rectangular box full of TIP3P water molecules (Jorgensen et al. 1983) with the box boundary extending at least $10 \AA$ Away from any solute atom. The Particle Mesh Ewald (PME) method (Darden et al. 1993) was used to calculate the full electrostatic energy of a unit cell in a macroscopic lattice of repeating images, SHAKE algorithm (Ryckaert et al. 1977) was employed to constrain covalent bonds involving hydrogen atoms. First, the system was gradually relaxed by 500 steps of steepest descent and 2500 steps of conjugate gradient (Yang et al. 2015, 2016). The MD simulations consisted of a gradual temperature increase from 0 to $300 \mathrm{~K}$ over 500 ps, a 2-ns simulation for equilibration and a 50-ns simulation for data collection, during which the 200 snapshots of complex dynamics were saved every 100 ps (Zhou et al. 2016).

Based on collected snapshots the total binding free energy $\Delta G_{\mathrm{ttl}}$ BD-I $/ \Delta G_{\mathrm{ttl}}$ BD-II of peptide ligand to Brd2 BD-I/ BD-II protein was calculated using molecular mechanics/ Poisson-Boltzmann surface area (MM/PBSA) (Homeyer and Gohlke 2012) and normal mode analysis (NMA) (Bahar et al. 2010), which can be decomposed into three components: intermolecular interaction energy $\left(\Delta E_{\text {int }}\right)$ between the protein and peptide, desolvation effect $\left(\Delta G_{\mathrm{dslv}}\right)$ and entropic penalty $(-T \Delta S)$ upon the binding (Yu et al. 2014). Here, the $\Delta E_{\mathrm{int}}$ and $\Delta G_{\mathrm{dslv}}$ were computed by MM/ PBSA using mmpbsa program, while the $-T \Delta S$ was estimated with NMA using nmode program. Due to the high computational demand, only 50 representative snapshots taken from MD trajectory were used to conduct NMA analysis, where frequencies of the vibrational modes were analyzed at $300 \mathrm{~K}$ for each snapshot using a harmonic approximation of the energies (Zhou et al. 2017). The mmpbsa and nmode are two build-in modules of AMBER suite; they are compatible with each other.

\section{Fluorescence polarization}

The acetylated peptides Stat3_K87 and Stat3(KGG)_K87 were prepared via Fmoc solid peptide chemistry and their $\mathrm{N}$-termini were labeled with rhodamine. The fluorescence polarization assays were described previously (Tyler et al. 2010; Zhu et al. 2017). Recombinant human Brd2 BD-I or $\mathrm{BD}-\mathrm{II}$ protein was added to the labeled peptide solution $(20 \mathrm{nM})$ in buffer containing in a buffer $25 \mathrm{mM}$ HEPES, $\mathrm{pH}$ $7.2,0.1 \%$ Tween 20 and 2 mM DTT. The data were corrected for background of the free labeled peptides. Each assay was performed in duplicate. The dissociation constants $\left(K_{\mathrm{d}}\right)$ were determined by fitting titration curves.

\section{Results and Discussion}

Structural and energetic analysis of acetylated peptides binding to Brd2 BD-I and BD-II

Histone acetylation code determines the recognition specificity of BET family proteins (Kanno et al. 2004). According to previous crystallographic and calorimetric studies acetylation at different lysine residues (Lys5, Lys8 and Lys12) of the $\mathrm{H} 4 \mathrm{~N}$-terminal tail would result in distinct specificity for the Brd2 BD-I and BD-II (Filippakopoulos et al. 2012; Marchand et al. 2015). Surface plasmon resonance assays revealed that the BD-I prefers to bind H4_K12 $\left(K_{\mathrm{d}}=930 \mu \mathrm{M}\right)$ as compared with H4_K5 $\left(K_{\mathrm{d}}=1800 \mu \mathrm{M}\right)($ Umehara et al. 2010b), whereas crystallographic analysis suggested that the BD-II possesses higher affinity for H4_K5 than H4_K12 (Umehara et al. 2010a). Diacetylation at Lys5 and Lys12 (H4_K5K12) can considerably improve the binding capability of BD-I and BD-II due to multiple-site recognition $\left(K_{\mathrm{d}}=360 \mu \mathrm{M}\right)$, while acetylation at Lys8 (H4_K8) appears to be unfavorable for both the two bromodomains (Umehara et al. 2010a).

Here, sequence alignment between human Brd2 BD-I (residues 91-163) and BD-II (residues 364-436) (UniProt: P25440) was carried out using MView program (Brown et al. 1998), revealing a high evolutionary conservation of the two homologous bromodomains (identity $=53.4 \%$ ) (Figure 1A). However, some residues seem to be variable over the two bromodomains; structural examination revealed that most of these variable residues locate at the loop regions around the protein active site, which are speculated to confer recognition specificity for different sequence patterns flanking the acetyl-lysine residues of H4 N-terminal tail. The crystal structures of BD-I in complex with H4_K12 peptide (PDB: 2DVQ) and BD-II in complex with $\mathrm{H} 4$ K5 512 peptide (PDB: 2E3K) were retrieved from the PDB database (Berman et al. 2000) and shown in Figure 1B. A distinct binding mode of the two complex systems can be readily observed: the whole 
H4_K12 peptide tightly binds on the surface of BD-I protein with its primary anchor Aly12 (acetylated Lys12) inserting deeply into the protein active site; only the Aly5 (acetylated Lys5) residue of H4_K5K12 can effectively interact with BD-II active site, while rest of the peptide is far away from the protein and exhibits large flexibility and intrinsic disorder. Crystallographic study also suggested that the positively charged Lys8 residue of H4_K12 peptide should play an important role in BD-I-peptide recognition by forming electrostatic attraction with a negatively charged cavity of BD-I (Umehara et al. 2010b). However, this residue seems to be marginal in BD-II-H4_K5K12 interaction since its side chain points out of BD-II surface and no effective nonbonded interactions are found with the protein (Figure $2 \mathrm{~B}$ ).

Next, the binding energetics of different acetylated $\mathrm{H} 4$ $\mathrm{N}$-terminal peptides to Brd2 BD-I and BD-II were calculated and decomposed using MM/PBSA and NMA based on the hundreds of bromodomain-peptide complex snapshots extracted from MD dynamics trajectory, including intermolecular interaction energy between bromodomain and peptide $\left(\Delta E_{\text {int }}\right)$, desolvation effect due to the bromodomain-peptide binding $\left(\Delta G_{\mathrm{dsl}}\right)$, and entropy penalty upon the binding $(-T \Delta S)$ as well as their sum, the total binding free
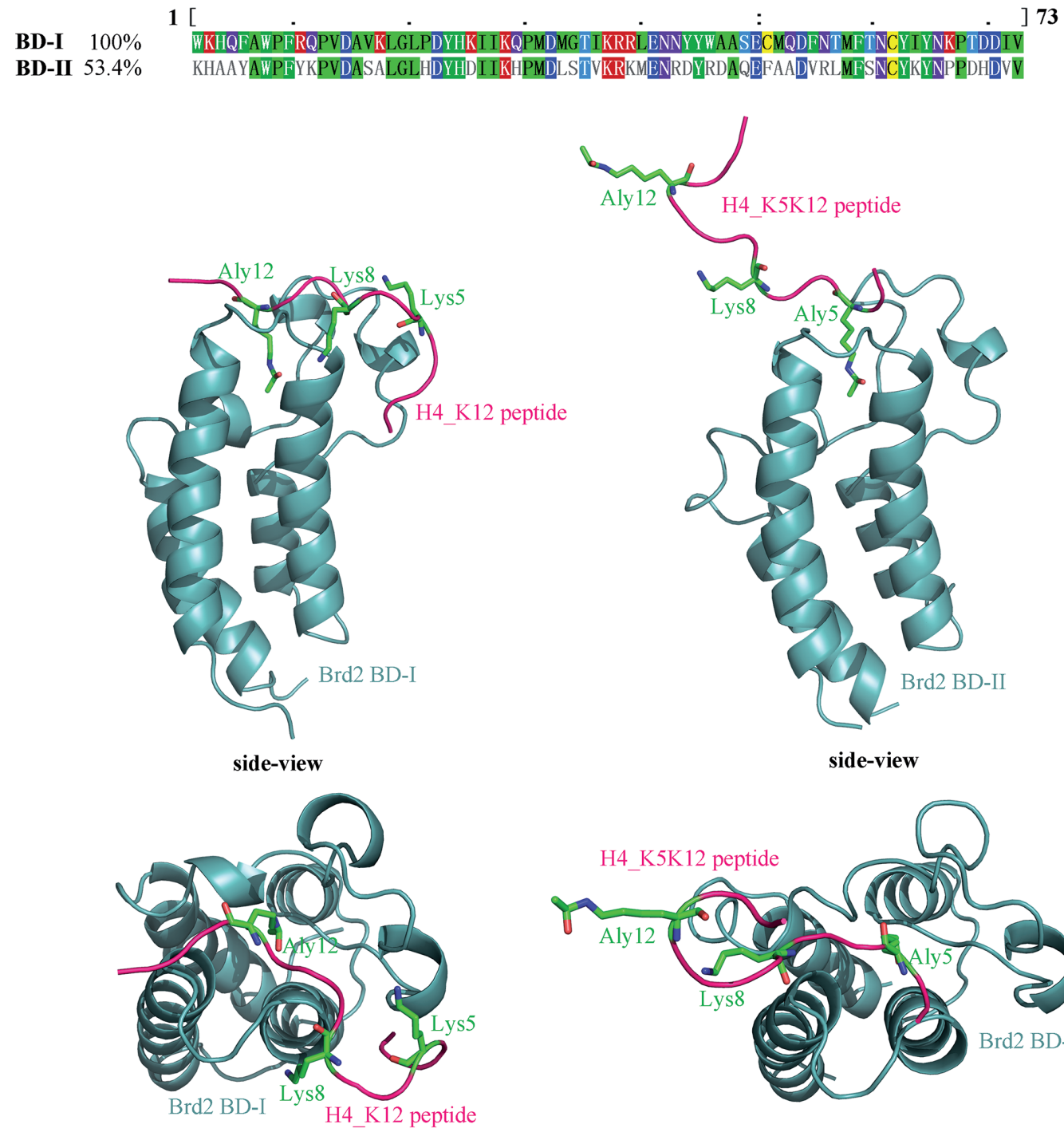

side-view

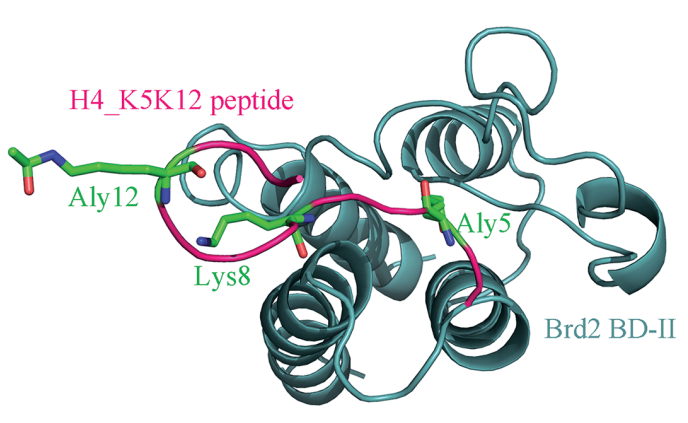

top-view

top-view

Figure 1. A. Sequence alignment between human Brd2 BD-I (residues 91-163) and BD-II (residues 364-436) (UniProt: P25440). B. Crystal complex structures of Brd2 BD-I with H4_K12 peptide (PDB: 2DVQ) and Brd2 BD-II with H4_K5K12 peptide (PDB: 2E3K). Cyan, protein; pink, peptide; green, residue. (See online version for color figure.) 
energy $\left(\Delta G_{\mathrm{ttl}}\right)$. In addition, peptide selectivity between the two bromodomains is characterized as $\Delta \Delta G_{\mathrm{ttl}}=\Delta G_{\mathrm{ttl}}$ BD-II $\Delta G_{\mathrm{ttl}}{ }^{\mathrm{BD}-\mathrm{I}}$ (Table 2). As can been seen, H4_K5 has generally a moderate affinity to both BD-I/BD-II $\left(\Delta G_{\mathrm{ttl}} \mathrm{BD}^{\mathrm{I}} / \Delta G_{\mathrm{ttl}} \mathrm{BD}-\mathrm{II}\right.$ $=-7.0 /-7.9 \mathrm{kcal} / \mathrm{mol})$ and low selectivity between the two bromodomains $\left(\Delta \Delta G_{\mathrm{ttl}}=-0.9 \mathrm{kcal} / \mathrm{mol}\right)$, but H4_K12 possesses high affinity to BD-I $\left(\Delta G_{\mathrm{ttl}}^{\mathrm{BD}-\mathrm{I}}=-12.5 \mathrm{kcal} / \mathrm{mol}\right)$ and considerable selectivity for BD-I over BD-II $\left(\Delta \Delta G_{\mathrm{ttl}}=\right.$ $3.9 \mathrm{kcal} / \mathrm{mol}$ ). Previously, Umehara et al. (2010b) demonstrated that the core recognition sequence of $\mathrm{H} 4 \mathrm{~N}$-terminal tail cover residues 6-15. As might be expected, the 10-mer acetylated peptide $\mathrm{cH} 4$ _K12 $\left({ }^{6} \mathrm{GGKGLGK} K_{a c} \mathrm{GGA}^{15}\right)$ exhibits a similar binding energetic profile $\left(\Delta G_{\mathrm{ttl}}{ }^{\mathrm{BD}-\mathrm{I}} / \Delta G_{\mathrm{ttl}} \mathrm{BD}-\mathrm{II}=\right.$ $-13.1 /-8.9 \mathrm{kcal} / \mathrm{mol}$ and $\left.\Delta \Delta G_{\mathrm{ttl}}=4.2 \mathrm{kcal} / \mathrm{mol}\right)$ with the full-length H4_K12 $\left(\Delta G_{\mathrm{ttl}}{ }^{\mathrm{BD}-\mathrm{I}} / \Delta G_{\mathrm{ttl}}{ }^{\mathrm{BD}-\mathrm{II}}=-12.5 /-8.6 \mathrm{kcal} /\right.$ mol and $S=3.9 \mathrm{kcal} / \mathrm{mol})$. In contrast, the Stat3_K87 peptide has a high affinity to both the two bromodomains $\left(\Delta G_{\mathrm{ttl}}\right.$ BD-I/ $\left.\Delta G_{\mathrm{ttl}}{ }^{\mathrm{BD}-\mathrm{II}}=-10.5 /-14.2 \mathrm{kcal} / \mathrm{mol}\right)$ but reversed selectivity for BD-II over BD-I $\left(\Delta \Delta G_{\mathrm{ttl}}=-3.7 \mathrm{kcal} / \mathrm{mol}\right)$. Recently, Zhu et al. (2017) found that the peptide is a good binder of $\mathrm{Brd} 2$ BD-II $\left(K_{\mathrm{d}}=5.9 \mu \mathrm{M}\right)$ and a moderate selector for Brd2 over Brd4 (2.5-fold). The difference in binding profile between $\mathrm{H} 4 \mathrm{~N}$-terminal peptide and Stat3 derived peptide imparts sequence and structural implications for rational design of acetylated peptide ligands with high affinity and selectivity. A further energetic decomposition revealed that these peptides have a very favorable interaction with bromodomains $\left(\Delta E_{\mathrm{int}}=\sim-60 \mathrm{kcal} / \mathrm{mol}\right)$, which, however, would be largely counteracted by the unfavorable solvent effect $\left(\Delta G_{\mathrm{dslv}}=\right.$ $\sim 30 \mathrm{kcalmol})$ and entropy penalty $(-T \Delta S=\sim 20 \mathrm{kcalmol})$, thus exhibiting a moderate binding capability to the bromo-

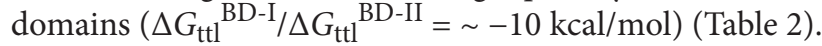
It is expected that the exquisite equilibrium among the flexibility, hydrophobicity and acetylation position would determine a peptide affinity and selectivity between the two

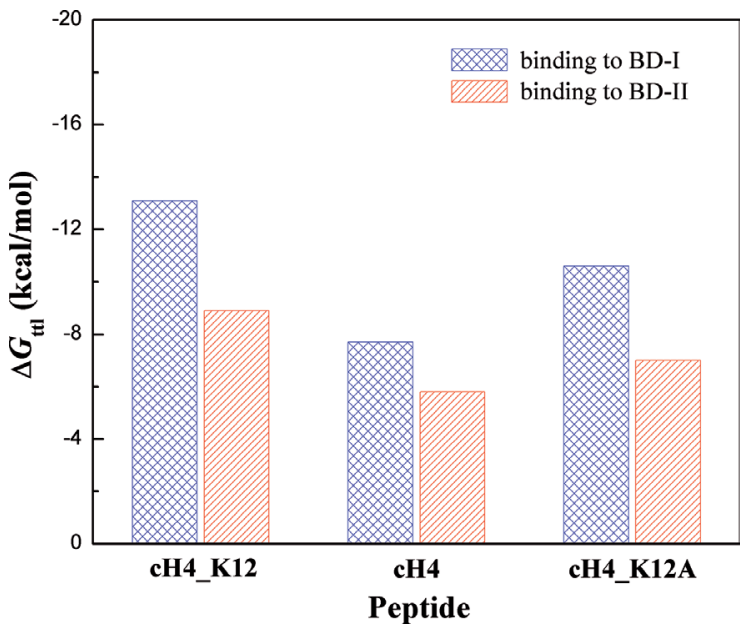

Figure 2. Binding free energies of $\mathrm{cH} 4$ K12 peptide as well as its deacetylated counterpart $\mathrm{cH} 4$ and $\mathrm{K} 12 \mathrm{~A}$ mutant $\mathrm{cH} 4$-K12A to Brd2 BD-I and BD-II.

bromodomains, which can carefully designed in a rational manner.

We calculated the binding free energies of $\mathrm{cH} 4 \_\mathrm{K} 12$ peptide $\left({ }^{6} \mathrm{GGKGLGK} K_{a c} \mathrm{GGA}^{15}\right)$ as well as its deacetylated counterpart (cH4, ${ }^{6}$ GGKGLGKGGA ${ }^{15}$ ) and K12A mutant (cH4_K12A, ${ }^{6}$ GGKGLGAGGA ${ }^{15}$ ), to Brd2 BD-I and BD-II. As shown in Figure 2, the deacetylation and K12A mutation would, respectively, considerably and moderately reduce peptide binding affinity. This is expected because the binding site of Brd2 bromodomains is a hydrophobic, deeply narrow pocket that can well accommodate the uncharged, long side-chain acetyl-lysine residue of $\mathrm{cH} 4$ K12 peptide. Deacetylation introduces a positive charge to the residue that is very unfavorable for the hydrophobic pocket, while K12A

Table 2. Calculated binding energetics of acetylated peptides binding to Brd2 BD-I and BD-II

\begin{tabular}{|c|c|c|c|c|c|c|}
\hline \multirow[b]{2}{*}{ Peptide } & \multirow[b]{2}{*}{ Bromodomain } & \multicolumn{5}{|c|}{ Binding energetics $(\mathrm{kcal} / \mathrm{mol})$} \\
\hline & & $\Delta E_{\text {int }}$ & $\Delta G_{\mathrm{dslv}}$ & $-T \Delta S$ & $\begin{array}{l}\Delta G_{\mathrm{ttl}}^{\mathrm{BD}-\mathrm{I}} / \\
\Delta G_{\mathrm{ttl}}^{\mathrm{BD}-\mathrm{II}}\end{array}$ & $\Delta \Delta G_{\mathrm{ttl}^{\prime}}^{a}$ \\
\hline \multirow{2}{*}{ H4_K5 } & BD-I & -64.8 & 34.1 & 23.7 & -7.0 & \multirow{2}{*}{-0.9} \\
\hline & BD-II & -64.0 & 31.5 & 24.6 & -7.9 & \\
\hline \multirow{2}{*}{ H4_K12 } & BD-I & -76.5 & 37.4 & 26.6 & -12.5 & \multirow{2}{*}{3.9} \\
\hline & BD-II & -67.7 & 35.0 & 24.1 & -8.6 & \\
\hline \multirow{2}{*}{ cH4_K12 } & BD-I & -60.1 & 29.8 & 17.2 & -13.1 & \multirow{2}{*}{4.2} \\
\hline & BD-II & -51.4 & 27.1 & 15.4 & -8.9 & \\
\hline \multirow{2}{*}{ Stat3_K87 } & BD-I & -72.3 & 50.6 & 11.2 & -10.5 & \multirow{2}{*}{-3.7} \\
\hline & BD-II & -81.9 & 54.2 & 13.5 & -14.2 & \\
\hline
\end{tabular}



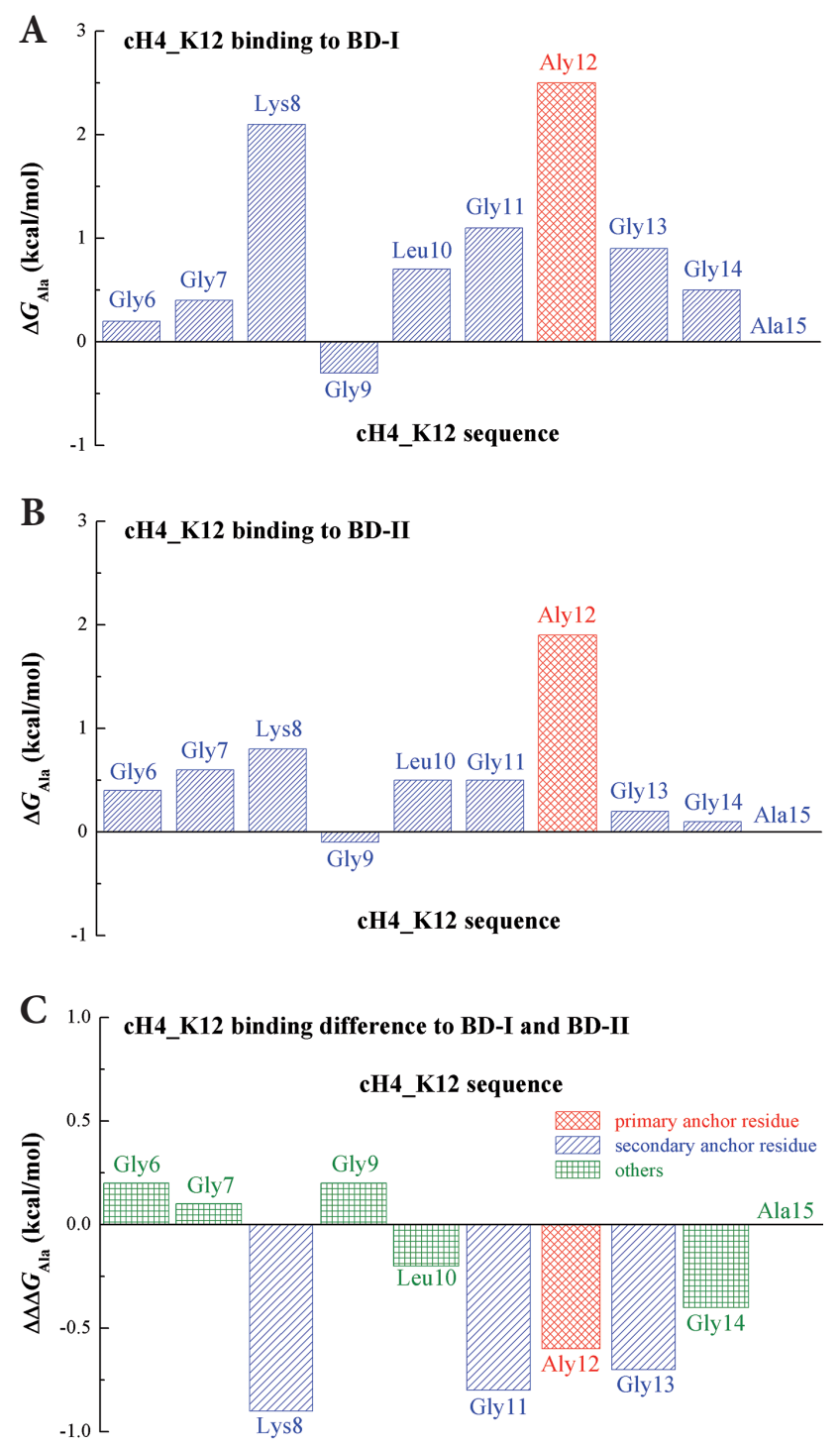

Figure 3. Relative residue importance of $\mathrm{cH} 4$ K12 peptide binding to Brd2 BD-I (A) and BD-II (B) as well as their difference (C), calculated via computational alanine-scanning (Kortemme et al. 2004). mutation replaces the bulky acetyl-lysine by a small alanine that cannot provide sufficient hydrophobic contact with the pocket. It is worth noting that, although the deacetylation and $\mathrm{K} 12 \mathrm{~A}$ mutation can reduce the peptide binding affinity to both the Brd2 BD-I and BD-II, they do not alter the peptide selectivity between the two bromodomains.

\section{Reshaping Stat3_K87 selectivity between Brd2 BD-I and $B D-I I$}

Computational alanine scanning (CAS) (Kortemme et al. 2004) was performed on the complexes of $\mathrm{CH} 4$ K12 peptide with $\mathrm{Brd} 2 \mathrm{BD}-\mathrm{I}$ and $\mathrm{BD}-\mathrm{II}$ to detect the relative residue importance of the peptide in the two complex systems. The scanning one-by-one mutated peptide residues to neutral alanine and then calculated total binding energy change $\left(\Delta G_{\mathrm{Ala}}\right)$ upon the mutation. The resulting $\Delta G_{\mathrm{Ala}}$ profiles for CH4_K12 binding to BD-I and BD-II are shown in Figure 3A and B, respectively. As can be seen, the two profiles are basically consistent, suggesting that the peptide can bind to the two bromodomains in a similar manner; most residues are favorable to bromodomain-peptide binding $\left(\Delta G_{\mathrm{Ala}}>0\right)$, while only few (e.g. Gly9) contribute unfavorably to the binding. As expected, the acetyl-lysine residue (Aly12) plays a crucial role in $\mathrm{CH} 4$-K12 interaction with both the two bromodomains $\left(\Delta G_{\mathrm{Ala}}=2.5\right.$ and $1.9 \mathrm{kcal} / \mathrm{mol}$ ), which is defined as the primary anchor and confers large stability to the two complex systems. In addition, the Lys8 residue is also important for BD-IcH4_K12 recognition $\left(\Delta G_{\mathrm{Ala}}>2 \mathrm{kcal} / \mathrm{mol}\right)$, whereas the residue seems not to be essential in $\mathrm{cH} 4$ _K12 binding to BD-II $\left(\Delta G_{\mathrm{Ala}}<1 \mathrm{kcal} / \mathrm{mol}\right)$.

The difference $\Delta \Delta G_{\text {Ala }}$ between the two profiles can straightforwardly characterize single residue contribution to peptide selectivity (Figure 3C). It is evident that the Lys8 has a considerable $\Delta \Delta G_{\mathrm{Ala}}=-0.9 \mathrm{kcal} / \mathrm{mol}$ versus the primary anchor Aly12 with a moderate $\Delta \Delta G_{\mathrm{Ala}}=-0.6 \mathrm{kcal} /$ mol, indicating that the Lys8 should play an important role

Table 3. The calculated binding energetics and experimental binding affinity of Stat3_K87 peptide and its grafted counterpart Stat3(KGG)_ K87 to Brd2 BD-I and BD-II

\begin{tabular}{|c|c|c|c|c|c|c|c|}
\hline \multirow{2}{*}{ Peptide } & \multirow{2}{*}{ Sequence } & \multicolumn{3}{|c|}{ Energy (kcal/mol) } & \multicolumn{3}{|c|}{ Affinity $(\mu \mathrm{M})$} \\
\hline & & $\Delta G_{\mathrm{ttl}}^{\mathrm{BD}-\mathrm{I}}$ & $\Delta G_{\mathrm{ttl}} \mathrm{BD}-\mathrm{II}$ & $\Delta \Delta G_{\mathrm{ttl}}^{a}$ & $K_{\mathrm{d}}^{\mathrm{BD}-\mathrm{I}}$ & $K_{\mathrm{d}}^{\mathrm{BD}-\mathrm{II}}$ & $S^{b}$ \\
\hline Stat3_K87 & ${ }^{281} \mathrm{HNLLRI}_{a c} \mathrm{QFLQS}^{292}$ & -10.5 & -14.2 & -3.7 & 46.2 & $9.7(5.9)^{c}$ & 0.21 -fold \\
\hline Stat3 & ${ }^{281}{ }^{\text {HNLLRIKQFLQS }}{ }^{292}$ & -5.8 & -8.7 & -3.3 & - & - & - \\
\hline Stat3_K87A & ${ }^{281}{ }^{\text {HNLLRIAQFLQS }}{ }^{292}$ & -7.6 & -12.9 & -2.9 & - & - & - \\
\hline Stat3(KGG)_K87 & ${ }^{281} \mathrm{HNKLRGK}_{a c}$ GFLQS $^{292}$ & -11.7 & -8.9 & 2.8 & 34.8 & 86.3 & 2.5-fold \\
\hline Stat3(K)_K87 & ${ }^{281} \mathrm{HNKLRI}_{a c} \mathrm{QFLQS}^{292}$ & -11.2 & -9.7 & 1.5 & - & - & - \\
\hline Stat3(G1)_K87 & ${ }^{281} \mathrm{HNLLRGK}_{a c} \mathrm{QFLQS}^{292}$ & -10.9 & -11.8 & -0.9 & - & - & - \\
\hline Stat3(G2)_K87 & ${ }^{281} \mathrm{HNLLRI}_{a c}$ GFLQS $^{292}$ & -10.4 & -12.1 & -1.7 & - & - & - \\
\hline
\end{tabular}

${ }^{a} \Delta \Delta G_{\mathrm{ttl}}=\Delta G_{\mathrm{ttl}}{ }^{\mathrm{BD}-\mathrm{II}}-\Delta G_{\mathrm{ttl}}{ }^{\mathrm{BD}-\mathrm{I}} ;{ }^{b} S=K_{\mathrm{d}}{ }^{\mathrm{BD}-\mathrm{II}} / K_{\mathrm{d}}{ }^{\mathrm{BD}-\mathrm{I}} ;{ }^{c}$ reported by Zhu et al. (2017). 
in peptide selectivity between the two bromodomains. In addition, two glycine residues Gly11 and Gly13 seem to be also important for the bromodomain-peptide recognition specificity $\left(\Delta \Delta G_{\mathrm{Ala}}=-0.8\right.$ and $-0.7 \mathrm{kcal} / \mathrm{mol}$, respectively); presence of glycine at these two positions can effectively improve peptide selectivity for BD-I over BD-II. This is in line with the fact that the Stat3_K87 peptide, a good BD-II binder, does not obey the requirement (i.e. $\mathrm{GK} \mathrm{Ka}_{\mathrm{ac}} \mathrm{G}$ in $\mathrm{cH} 4$ - $\mathrm{K} 12$ are replaced by $I K_{\mathrm{ac}} \mathrm{Q}$ in Stat3_K87, respectively). In this respect, we defined the Lys8, Gly11 and Gly13 as secondary anchor residues, which confer to peptide specificity in contrast to the primary anchor residue Aly12 that contributes to peptide affinity.

According to above analysis, it is found that the presence of Lys, Gly and Gly at the secondary anchor residues 8,11 and 13 , respectively, can effectively improve the $\mathrm{H} 4$ N-terminal peptide (H4_K12 or cH4_K12) selectivity for BD-I over BD-II. On the other hand, the Stat3_K87 peptide is a strong binder of $\mathrm{Brd} 2$ but it exhibits a higher affinity for BD-II than BD-I. Therefore, we considered to graft the three secondary anchor residues (Lys8, Gly11 and Gly13) of $\mathrm{H} 4 \mathrm{~N}$-terminal peptide to the three corresponding residue positions of Stat3_K87 $\left({ }^{281} \mathrm{HNLLRIK} a c\right.$ QFLQS $\left.{ }^{292}\right)$ to obtain its grafted counterpart Stat3(KGG)_K87 $\left({ }^{281}\right.$ HNKL$\mathrm{RGK}_{a c} \mathrm{GFLQS}^{292}$ ). The total binding energies of Stat3_K87 and Stat3(KGG)_K87 to BD-I and BD-II $\left(\Delta G_{t t l}{ }^{B D-I}\right.$ and $\Delta G_{\mathrm{ttl}}{ }^{\mathrm{BD}-\mathrm{II}}$ ) were calculated and compared in Table 3. As expected, the grafting can totally reverse peptide selectivity from the BD-II-over-BD-I type of native Stat3_K87 $\left(\Delta \Delta G_{\mathrm{ttl}}=-3.7 \mathrm{kcal} / \mathrm{mol}<0\right)$ to the BD-I-over-BD-II type of mutant Stat3(KGG)_K87 $\left(\Delta \Delta G_{\mathrm{ttl}}=2.8 \mathrm{kcal} / \mathrm{mol}>0\right)$, although the mutant binding capability seems to be degenerated as compared to native. In order to substantiate the computational grafting, binding affinities of Stat3_K87 and Stat3(KGG)_K87 to the two bromodomains $\left(K_{\mathrm{d}}^{\mathrm{BD}-\mathrm{I}}\right.$ and $K_{\mathrm{d}}^{\mathrm{BD}-\mathrm{II}}$ ) were determined using fluorescence polarization assays (Figure 4 and Table 3). As can be seen, Stat3_K87 can tightly bind to BD-II $\left(K_{\mathrm{d}}=9.7 \mu \mathrm{M}\right)$ - this is basically in line with a previous report $\left(K_{\mathrm{d}}=5.9 \mu \mathrm{M}\right)(\mathrm{Zhu}$ et al. 2017), but has only a moderate affinity to BD-I $\left(K_{\mathrm{d}}=46.2 \mu \mathrm{M}\right)$, thus displaying a BD-II-over-BD-I selectivity with $S=K_{\mathrm{d}}{ }^{\mathrm{BD}-\mathrm{II}} /$ $K_{\mathrm{d}}^{\mathrm{BD}-\mathrm{I}}=0.21$-fold (i.e. $K_{\mathrm{d}}{ }^{\mathrm{BD}-\mathrm{I}} / K_{\mathrm{d}}{ }^{\mathrm{BD}-\mathrm{II}}=4.8$-fold). In contrast, the affinity values of Stat3(KGG)_K87 to BD-I and BD-II were measured as 34.8 and $86.3 \mu \mathrm{M}$, respectively, and $S=K_{\mathrm{d}}^{\mathrm{BD}-\mathrm{II}} / K_{\mathrm{d}}^{\mathrm{BD}-\mathrm{I}}=2.5$-fold, suggesting that it is a typical BD-I-over-BD-II selective peptide). Both the computational and experimental investigations demonstrated that the acetylated peptide selectivity between BD-I and BD-II can be systematically changed by rationally designing and grafting few secondary anchor residues. In next works, we consider to finely tune peptide selectivity by exquisitely optimizing the amino acid types and their combination at the secondary anchor residues.

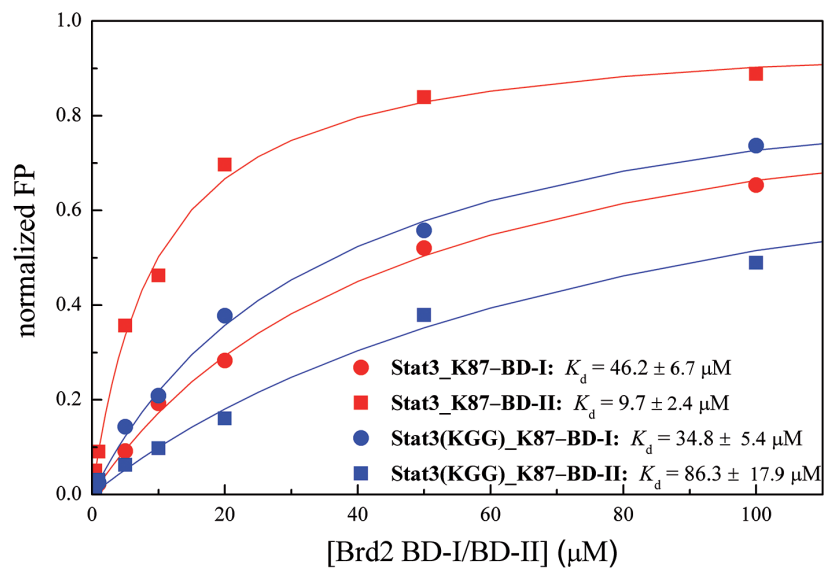

Figure 4. Determination of affinity for acetylated peptides Stat3 K87 and Stat3(KGG)_K87 binding to Brd2 BD-I and BD-II using fluorescence polarization (FP) assays.

Acknowledgements. This study was supported by the Suzhou Clinical Key Disease Diagnosis and Treatment Technology Foundation (No. LCZX201322), the Youth Medical Talents Project of Jiangsu (No. QNRC2016217), the Natural Science Foundation of China (No. 81302196), and the Natural Science Foundation of Suzhou (No. SYS201307).

Conflict of interest The authors declare no conflict of interest.

\section{References}

Bahar I, Lezon TR, Bakan A, Shrivastava IH (2010): Normal mode analysis of biomolecular structures: functional mechanisms of membrane proteins. Chem. Rev. 110, 1463-1497 https://doi.org/10.1021/cr900095e

Bai Z, Hou S, Zhang S, Li Z, Zhou P (2017): Targeting self-binding peptides as a novel strategy to regulate protein activity and function: a case study on the proto-oncogene tyrosine protein kinase c-Src. J. Chem. Inf. Model. 57, 835-845 https://doi.org/10.1021/acs.jcim.6b00673

Berman HM, Westbrook J, Feng Z, Gilliland G, Bhat TN, Weissig H, Shindyalov IN, Bourne PE (2000): The protein data bank. Nucl. Acids Res. 28, 235-242

https://doi.org/10.1093/nar/28.1.235

Berenguer-Daizé C, Astorgues-Xerri L, Odore E, Cayol M, Cvitkovic E, Noel K, Bekradda M, MacKenzie S, Rezai K, Lokiec F, et al. (2016): OTX015 (MK-8628), a novel BET inhibitor, displays in vitro and in vivo antitumor effects alone and in combination with conventional therapies in glioblastoma models. Int. J. Cancer 139, 2047-2055 https://doi.org/10.1002/ijc.30256

Bhattacharya D, Nowotny J, Cao R, Cheng J (2016): 3Drefine: an interactive web server for efficient protein structure refinement. Nucl. Acids Res. 44, W406-W409 https://doi.org/10.1093/nar/gkw336 
Brown NP, Leroy C, Sander C (1998): MView: a web-compatible database search or multiple alignment viewer. Bioinformatics 14, 380-381 https://doi.org/10.1093/bioinformatics/14.4.380

Case DA, Cheatham TE, Darden T, Gohlke H, Luo R, Merz KM, Onufriev A, Simmerling C, Wang B, Woods RJ (2005): The amber biomolecular simulation programs. J. Comput. Chem. 26, 1668-1688 https://doi.org/10.1002/jcc.20290

Cheng Z, Gong Y, Ma Y, Lu K, Lu X, Pierce LA, Thompson RC, Muller S, Knapp S, Wang J (2013): Inhibition of BET bromodomain targets genetically diverse glioblastoma. Clin. Cancer Res. 19, 1748-1759 https://doi.org/10.1158/1078-0432.CCR-12-3066

Cheung KL, Zhang F, Jaganathan A, Sharma R, Zhang Q, Konuma T, Shen T, Lee JY, Ren C, Chen CH, et al. (2017): Distinct roles of Brd2 and Brd4 in potentiating the transcriptional program for Th17 cell differentiation. Mol. Cell 65, 1-13 https://doi.org/10.1016/j.molcel.2016.12.022

Darden T, York D, Pedersen L (1993): Particale mesh Ewald and $\mathrm{N} . \log (\mathrm{N})$ method for Ewald sums in large systems. J. Chem. Phys. 98, 10089-10092

https://doi.org/10.1063/1.464397

Deeney JT, Belkina AC, Shirihai OS, Corkey BE, Denis GV (2016) BET Bromodomain proteins Brd2, Brd 3 and Brd4 selectively regulate metabolic pathways in the pancreatic $\beta$-cell. PLoS One 11, e0151329 https://doi.org/10.1371/journal.pone.0151329

Duan Y, Wu C, Chowdhury S, Lee MC, Xiong G (2003): A point charge force field for molecular mechanics simulations of proteins. J. Comput. Chem. 24, 1999-2012 https://doi.org/10.1002/jcc.10349

Filippakopoulos P, Qi J, Picaud S, Shen Y, Smith WB, Fedorov O, Morse EM, Keates T, Hickman TT, Felletar I, et al. (2010): Selective inhibition of BET bromodomains. Nature 468, 1067-1073 https://doi.org/10.1038/nature09504

Filippakopoulos P, Picaud S, Mangos M, Keates T, Lambert JP, Barsyte-Lovejoy D, Felletar I, Volkmer R, Müller S, Pawson T, et al. (2012): Histone recognition and large-scale structural analysis of the human bromodomain family. Cell 149, 214-231 https://doi.org/10.1016/j.cell.2012.02.013

Homeyer N, Gohlke H (2012): Free energy calculations by the molecular mechanics Poisson-Boltzmann surface area method. Mol. Inf. 31, 114-122 https://doi.org/10.1002/minf.201100135

Huang H, Zhang J, Shen W, Wang X, Wu J, Wu J, Shi Y (2007): Solution structure of the second bromodomain of $\mathrm{Brd} 2$ and its specific interaction with acetylated histone tails. BMC Struct. Biol. 7, 57 https://doi.org/10.1186/1472-6807-7-57

Jorgensen WL, Chandrasekhar J, Madura JD, Impey RW, Klein ML (1983): Comparison of simple potential functions for simulating liquid water. J. Phys. Chem. 79, 926-935 https://doi.org/10.1063/1.445869

Jung M, Gelato KA, Fernández-Montalván A, Siegel S, Haendler B (2015): Targeting BET bromodomains for cancer treatment. Epigenomics 7, 487-501 https://doi.org/10.2217/epi.14.91
Kanno T, Kanno Y, Siegel RM, Jang MK, Lenardo MJ, Ozato K (2004): Selective recognition of acetylated histones by bromodomain proteins visualized in living cells. Mol. Cell. 13, 33-43 https://doi.org/10.1016/S1097-2765(03)00482-9

Kortemme T, Kim DE, Baker D (2004): Computational alanine scanning of protein-protein interfaces. Sci STKE 2004, pl2 https://doi.org/10.1126/stke.2192004pl2

Khoury GA, Thompson JP, Smadbeck J, Kieslich CA, Floudas CA (2013): Forcefield_PTM: ab initio charge and AMBER forcefield parameters for frequently occurring post-translational modifications. J. Chem. Theory Comput. 9, 5653-5674 https://doi.org/10.1021/ct400556v

Lee DU, Katavolos P, Palanisamy G, Katewa A, Sioson C, Corpuz J, Pang J, DeMent K, Choo E, Ghilardi N, et al. (2016): Nonselective inhibition of the epigenetic transcriptional regulator BET induces marked lymphoid and hematopoietic toxicity in mice. Toxicol. Appl. Pharmacol. 300, 47-54 https://doi.org/10.1016/j.taap.2016.03.013

Li Y, Li M, Min W, Han G, Wang L, Chen C, Li Z, Zhang Y, Li J, Yue $Z$ (2017): Integrated in silico-in vitro characterization, identification and disruption of the intermolecular interaction between $\mathrm{SH} 3$ domain-containing protein kinases and human pituitary tumor-transforming gene 1. Gen. Physiol. Biophys. 36, 91-98 https://doi.org/10.4149/gpb_2016035

Long J, Li B, Rodriguez-Blanco J, Pastori C, Volmar CH, Wahlestedt C, Capobianco A, Bai F, Pei XH, Ayad NG, Robbins DJ (2014): The BET bromodomain inhibitor I-BET151 acts downstream of smoothened protein to abrogate the growth of hedgehog protein-driven cancers. J. Biol. Chem. 289, 35494-35502 https://doi.org/10.1074/jbc.M114.595348

Luo H, Du T, Zhou P, Yang L, Mei H, Ng H, Zhang W, Shu M, Tong W, Shi L, Mendrick DL, Hong H (2015): Molecular docking to identify associations between drugs and class I human leukocyte antigens for predicting idiosyncratic drug reactions. Comb. Chem. High Throughput Screen. 18, 296-304 https://doi.org/10.2174/1386207318666150305144015

Marchand JR, Caflisch A (2015): Binding mode of acetylated histones to bromodomains: variations on a common motif. ChemMedChem 10, 1327-1333 https://doi.org/10.1002/cmdc.201500141

Padmanabhan B, Mathur S, Manjula R, Tripathi S (2016): Bromodomain and extra-terminal (BET) family proteins: new therapeutic targets in major diseases. J. Biosci. 41, 295-311 https://doi.org/10.1007/s12038-016-9600-6

Pastori C, Daniel M, Penas C, Volmar CH, Johnstone AL, Brothers SP, Graham RM, Allen B, Sarkaria JN, Komotar RJ, et al. (2014): BET bromodomain proteins are required for glioblastoma cell proliferation. Epigenetics 9, 611-620 https://doi.org/10.4161/epi.27906

Picaud S, Wells C, Felletar I, Brotherton D, Martin S, Savitsky P, Diez-Dacal B, Philpott M, Bountra C, Lingard H, et al. (2013): RVX-208, an inhibitor of BET transcriptional regulators with selectivity for the second bromodomain. Proc. Natl. Acad. Sci. USA 11, 19754-19759 https://doi.org/10.1073/pnas.1310658110

Ryckaert J, Ciccotti G, Berendsen HJC (1977): Numerical integration of Cartesian equations of motion of a system with 
constraints - molecular dynamics of n-alkanes. J. Comput. Phys. 23, 327-341 https://doi.org/10.1016/0021-9991(77)90098-5

Ryu J, Lee M, Cha J, Laskowski RA, Ryu SE, Kim DS (2016): BetaSCPWeb: side-chain prediction for protein structures using Voronoi diagrams and geometry prioritization. Nucl. Acids Res. 44, W416-W423 https://doi.org/10.1093/nar/gkw368

Taniguchi Y (2016): The bromodomain and extra-terminal domain (BET) family: functional anatomy of BET paralogous proteins. Int. J. Mol. Sci. 17, E1849 https://doi.org/10.3390/ijms17111849

Tian F, Lv Y, Zhou P, Yang L (2011): Characterization of PDZ domain-peptide interactions using an integrated protocol of QM/MM, PB/SA, and CFEA analyses. J. Comput. Aided Mol. Des. 25, 947-958 https://doi.org/10.1007/s10822-011-9474-5

Tsunaka Y, Kajimura N, Tate S, Morikawa K (2005): Alteration of the nucleosomal DNA path in the crystal structure of a human nucleosome core particle. Nucl. Acids Res. 33, 3424-3434 https://doi.org/10.1093/nar/gki663

Tyler RC, Peterson FC, Volkman BF (2010): Distal interactions within the par3-VE-cadherin complex. Biochemistry 49, 951-957 https://doi.org/10.1021/bi9017335

Umehara T, Nakamura Y, Wakamori M, Ozato K, Yokoyama S, Padmanabhan B (2010a): Structural implications for K5/K12di-acetylated histone $\mathrm{H} 4$ recognition by the second bromodomain of BRD2. FEBS Lett. 584, 3901-3908 https://doi.org/10.1016/j.febslet.2010.08.013

Umehara T, Nakamura Y, Jang MK, Nakano K, Tanaka A, Ozato K, Padmanabhan B, Yokoyama S (2010b): Structural basis for acetylated histone $\mathrm{H} 4$ recognition by the human BRD2 bromodomain. J. Biol. Chem. 285, 7610-7618
https://doi.org/10.1074/jbc.M109.062422

Yang C, Zhang S, He P, Wang C, Huang J, Zhou P (2015): Selfbinding peptides: folding or binding? J. Chem. Inf. Model. $55,329-342$ https://doi.org/10.1021/ci500522v

Yang C, Zhang S, Bai Z, Hou S, Wu D, Huang J, Zhou P (2016): A two-step binding mechanism for the self-binding peptide recognition of target domains. Mol. Biosyst. 12, 1201-1213 https://doi.org/10.1039/C5MB00800J

Yu H, Zhou P, Deng M, Shang Z (2014): Indirect readout in protein-peptide recognition: a different story from classical biomolecular recognition. J. Chem. Inf. Model. 54, 2022-2032

https://doi.org/10.1021/ci5000246

Zhou P, Zhang S, Wang Y, Yang C, Huang J (2016): Structural modeling of HLA-B*1502/peptide/carbamazepine/T-cell receptor complex architecture: implication for the molecular mechanism of carbamazepine-induced Stevens-Johnson syndrome/toxic epidermal necrolysis. J. Biomol. Struct. Dyn. 34, 1806-1817 https://doi.org/10.1080/07391102.2015.1092476

Zhou P, Hou S, Bai Z, Li Z, Wang H, Chen Z, Meng Y (2017): Disrupting the intramolecular interaction between protooncogene c-Src SH3 domain and its self-binding peptide PPII with rationally designed peptide ligands. Artif. Cells Nanomed. Biotechnol. 45, 1-10

Zhu L, Ding X (2018): Molecular design of Stat3-derived peptide selectivity between BET proteins $\mathrm{Brd} 2$ and $\mathrm{Brd} 4$ in ovarian cancer. J. Mol. Recog. 31, e2679 https://doi.org/10.1002/jmr.2679

Received: November 17, 2017

Final version accepted: December 29, 2017

First published online: June 29, 2018 